\title{
Ferromagnetic Phase Transition for the Spanning-Forest Model $(q \rightarrow 0$ Limit of the Potts Model) in Three or More Dimensions
}

\author{
Youjin Deng, ${ }^{1, *}$ Timothy M. Garoni, ${ }^{1, \dagger}$ and Alan D. Sokal ${ }^{1,2, \pitchfork}$ \\ ${ }^{1}$ Department of Physics, New York University, 4 Washington Place, New York, New York 10003, USA \\ ${ }^{2}$ Department of Mathematics, University College London, London WC1E 6BT, United Kingdom
}

(Received 6 October 2006; published 17 January 2007)

\begin{abstract}
We present Monte Carlo simulations of the spanning-forest model ( $q \rightarrow 0$ limit of the ferromagnetic Potts model) in spatial dimensions $d=3,4,5$. We show that, in contrast to the two-dimensional case, the model has a ferromagnetic second-order phase transition at a finite positive value $w_{c}$. We present numerical estimates of $w_{c}$ and of the thermal and magnetic critical exponents. We conjecture that the upper critical dimension is 6 .
\end{abstract}

DOI: 10.1103/PhysRevLett.98.030602

The Potts model [1,2] plays an important role in the modern theory of phase transitions and critical phenomena and is characterized by two parameters: the number $q$ of Potts spin states, and the nearest-neighbor coupling $v=$ $e^{\beta J}-1$. Initially $q$ is a positive integer and $v$ is a real number in the interval $-1 \leq v<+\infty$, but the FortuinKasteleyn (FK) representation [3] shows that the partition function $Z_{G}(q, v)$ of the $q$-state Potts model on any finite graph $G$ is in fact a polynomial in $q$ and $v$. This allows us to interpret $q$ and $v$ as taking arbitrary real or even complex values and to study the phase diagram of the Potts model in the real $(q, v)$ plane or in complex $(q, v)$ space. In particular, when $q, v>0$ the FK representation has positive weights and hence can be interpreted probabilistically as a correlated bond-percolation model: the FK randomcluster model [4]. In this way we can study all positive values of $q$, integer or noninteger, within a unified framework.

In two dimensions, the behavior of the ferromagnetic $(v>0)$ Potts/random-cluster model is fairly well understood, thanks to a combination of exact solutions [5], Coulomb-gas methods [6], and conformal field theory [7]. But in dimension $d \geq 3$, many important aspects remain unclear: the location of the crossover between second-order and first-order behavior [8]; the nature of the critical exponents and their dependence on $q$; the value of the upper critical dimension for noninteger $q$; and the qualitative behavior of the critical curve $v_{c}(q)$ near $q=0$.

Interesting special cases of the random-cluster model arise in the limit $q \rightarrow 0$. In particular, the limit $q, v \rightarrow 0$ with $w=v / q$ held fixed gives rise to a model of spanning forests, i.e., spanning subgraphs without cycles, in which each occupied edge gets a weight $w$ [9]. Very recently, it was shown [10] - generalizing Kirchhoff's matrix-tree theorem [11] - that this spanning-forest model can be mapped onto a fermionic (Grassmann) theory involving a quadratic (Gaussian) term and a special nearest-neighbor four-fermion term. Moreover, this fermionic model possesses an $\operatorname{OSP}(1 \mid 2)$ supersymmetry and can be mapped, to all orders of the perturbation theory in powers of $1 / w$, onto
PACS numbers: 05.50.+q, 11.10.Kk, 64.60.Cn, 64.60.Fr

an $N$-vector model [O $(N)$-invariant $\sigma$ model] analytically continued to $N=-1$. It follows that, in two dimensions, the spanning-forest model is perturbatively asymptotically free, in close analogy to (large classes of) two-dimensional $\sigma$ models and four-dimensional non-Abelian gauge theories. In particular, the only ferromagnetic $(w>0)$ critical point lies at $w_{c}=+\infty$, in agreement [12] with the exact solutions on the square, triangular, and hexagonal lattices [5] showing that $v_{c}(q) \propto q^{1 / 2}$ as $q \downarrow 0$.

In this Letter we study the spanning-forest model in spatial dimensions $d \geq 3$, using Monte Carlo methods. We will show that, in contrast to the two-dimensional case, the model has a "ferromagnetic" second-order phase transition at a finite positive value $w_{c}$, and we will estimate the thermal and magnetic critical exponents as well as a universal amplitude ratio. It follows that $v_{c}(q) \propto q$ as $q \downarrow 0$. Indeed, we see the present study of the spanning-forest model as the first step in a comprehensive study of the random-cluster model as a function of (noninteger) $q$.

For the random-cluster model with $q \geq 1$, a collectivemode Monte Carlo algorithm has recently been invented by Chayes and Machta [13]; it generalizes the well-known Swendsen-Wang algorithm [14] and reduces to (a slight variant of) it when $q$ is an integer. But for $q<1$, the only available algorithm seems to be the Sweeny algorithm [15], which is a local bond-update algorithm. Ordinarily one would expect such a local algorithm to exhibit severe critical slowing-down, at least when the specific heat is divergent [16]. But the random-cluster model with $q<$ $q_{0}(d) \approx 2$ has a nondivergent specific heat (i.e., critical exponent $\alpha<0$ ), which suggests that the critical slowingdown might not be so severe after all. Indeed, our numerical studies of the spanning-forest model (i.e., the $q \rightarrow 0$ limit) in dimensions $d=2,3,4,5$ strongly suggest that there is no critical slowing-down; i.e., the dynamic critical exponent $z_{\exp }$ associated with the exponential autocorrelation time is zero. Better yet, the exponent $z_{\text {int, } \mathcal{O}}$ associated with the integrated autocorrelation time [17] turns out to be negative for "global" observables such as the mean-square cluster size; that is, one "effectively independent" sample 


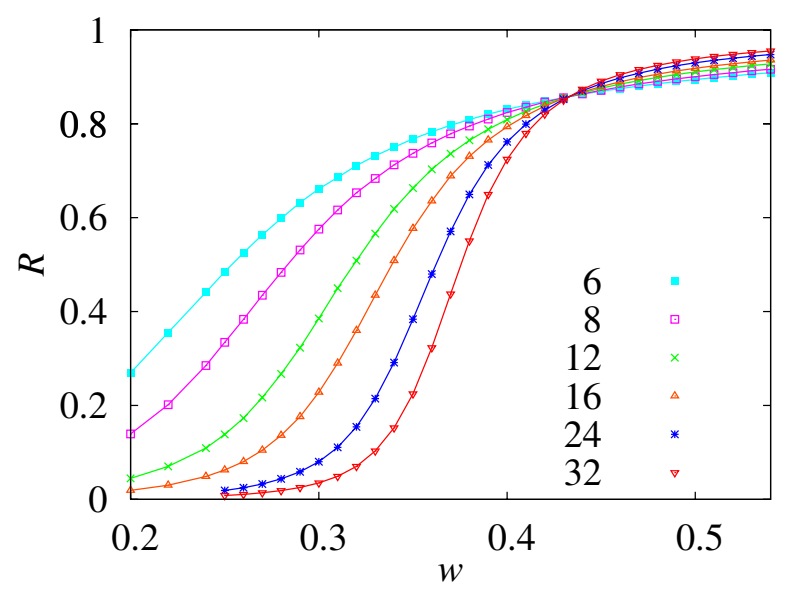

FIG. 1 (color online). Coarse plot of $R$ vs $w$ for spanning forests in dimension $d=3$ and lattice sizes $6 \leq L \leq 32$.

can be obtained in a time much less than a single "sweep" — a kind of "critical speeding-up."

On the other hand, the Sweeny algorithm for $q \neq 1$ requires a nonlocal connectivity check each time one tries to update a single bond. If done in the naive way (e.g., by depth-first or breadth-first search), this would require a CPU time of the order of the mean cluster size $\chi \propto L^{\gamma / \nu}=$ $L^{\approx 2}$ per "hit" of a single bond, leading to a severe "computational critical slowing-down." Recent work by computer scientists on dynamic connectivity algorithms [18] shows how this can be reduced to $(\log L)^{p}$, but at the expense of fairly complicated algorithms and data structures. We therefore adopted an intermediate solution: a simple "homemade" dynamic connectivity algorithm that empirically has a slowing-down $L^{\approx 0.7}$. The details of this algorithm, along with measurements of the dynamic critical behavior of the Sweeny algorithm in the spanningforest limit, will be reported separately [19].

We simulated the spanning-forest model in dimensions $d=3,4,5$ on hypercubic lattices of size $L^{d}$ with periodic boundary conditions. We measured the cluster-size moments $\mathcal{S}_{k}=\sum_{\text {clusters } \#(\mathcal{C})^{k}}$ for $k=0,2$, 4. We focused attention on the ratio $R=\left\langle\mathcal{S}_{4}\right\rangle /\left\langle\mathcal{S}_{2}^{2}\right\rangle$, which tends in the infinite-volume limit to 0 in a disordered phase and to 1 in an ordered phase, and is therefore diagnostic of a phase transition. We also studied $\left\langle\mathcal{S}_{2}\right\rangle$ in order to estimate the magnetic critical exponent.

In each dimension, we began by making a "coarse" set of runs covering a wide range of $w$ values, using modestsized lattices and modest statistics. If the plots of $R$ vs $w$ indicated a likely phase transition, we then made a "fine" set of runs covering a small neighborhood of the estimated critical point, using larger lattices and larger statistics. Finally, using the results from these latter runs, we made a "superfine" set of runs extremely close to the estimated critical point, using as large lattices and statistics as we could manage, with the goal of obtaining precise quantitative estimates of the critical point $w_{c}$ and the critical

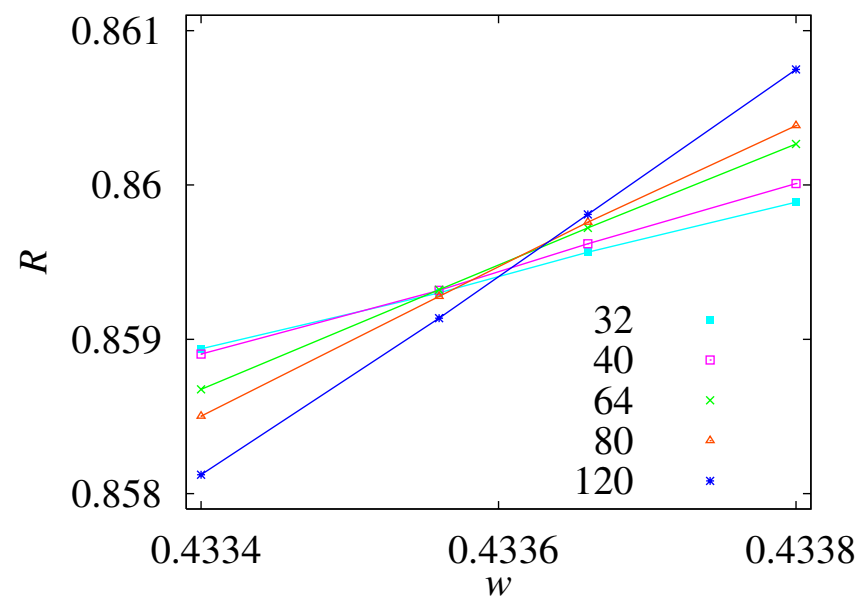

FIG. 2 (color online). " "Superfine" plot of $R$ vs $w$ for spanning forests in dimension $d=3$ and lattice sizes $32 \leq L \leq 120$.

exponents. The complete set of runs reported in this Letter used approximately $7 \mathrm{yr} \mathrm{CPU}$ time on a $3.2 \mathrm{GHz}$ Xeon EM64T processor.

The coarse plot of $R$ vs $w$ for dimension $d=3$ and lattice sizes $6 \leq L \leq 32$ is shown in Fig. 1, and shows a clear order-disorder transition at $w_{c} \approx 0.43$. The corresponding superfine plot, for lattice sizes $32 \leq L \leq 120$, is shown in Fig. 2. We fit the data to Ansätze obtained from

$$
\begin{aligned}
R= & R_{c}+a_{1}\left(w-w_{c}\right) L^{1 / \nu}+a_{2}\left(w-w_{c}\right)^{2} L^{2 / \nu} \\
& +b_{1} L^{-\omega_{1}}+b_{2} L^{-\omega_{2}}+\cdots
\end{aligned}
$$

by omitting various subsets of terms, and we systematically varied $L_{\min }$ (the smallest $L$ value included in the fit). We also made analogous fits for $\left\langle\mathcal{S}_{2}\right\rangle / L^{\gamma / \nu}$. Comparing all these fits, we estimate the critical point $w_{c}=0.43365 \pm$ 0.00002 , the critical exponents $\nu=1.28 \pm 0.04$ and $\gamma / \nu=2.1675 \pm 0.0010$, and the universal amplitude ratio

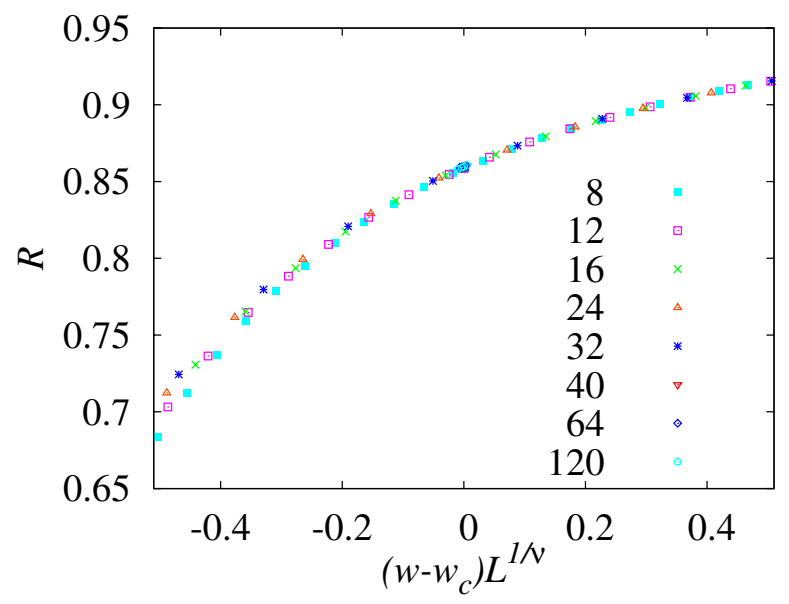

FIG. 3 (color online). Finite-size-scaling plot of $R$ vs $(w-$ $\left.w_{c}\right) L^{1 / \nu}$, with $w_{c}=0.43365$ and $\nu=1.28$, for spanning forests in dimension $d=3$ and lattice sizes $8 \leq L \leq 120$. 


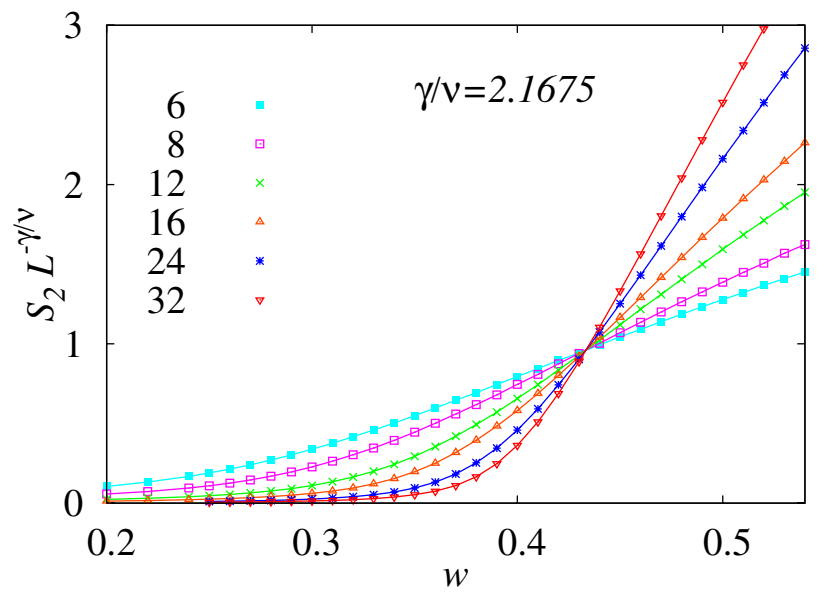

FIG. 4 (color online). Plot of $\left\langle\mathcal{S}_{2}\right\rangle / L^{\gamma / \nu}$ vs $w$, with $\gamma / \nu=$ 2.1675 , for spanning forests in dimension $d=3$ and lattice sizes $6 \leq L \leq 32$.

$R_{c}=0.8598 \pm 0.0003(68 \%$ subjective confidence intervals, including both statistical error and estimated systematic error due to unincluded corrections to scaling). A finite-size-scaling plot using these parameters is shown in Fig. 3. A coarse plot of $\left\langle\mathcal{S}_{2}\right\rangle / L^{\gamma / \nu}$ using the estimated value of $\gamma / \nu$ is shown in Fig. 4 .

The coarse plots of $R$ vs $w$ for dimensions $d=4,5$ are shown in Figs. 5 and 6, respectively. Once again they show a clear order-disorder transition. For lack of space, we refrain from showing the corresponding superfine plots (which use lattice sizes up to $64^{4}$ and $20^{5}$ ) and simply give the results of fits to Ansätze of the general type (1). In dimension $d=4$, we estimate $w_{c}=0.210302 \pm$ $0.000010, \quad \nu=0.80 \pm 0.01, \quad \gamma / \nu=2.1603 \pm 0.0010$, and $R_{c}=0.73907 \pm 0.00010$. In dimension $d=5$, we estimate $\quad w_{c}=0.14036 \pm 0.00002, \quad \nu=0.59 \pm 0.02$, $\gamma / \nu=2.08 \pm 0.02$, and $R_{c}=0.625 \pm 0.015$.

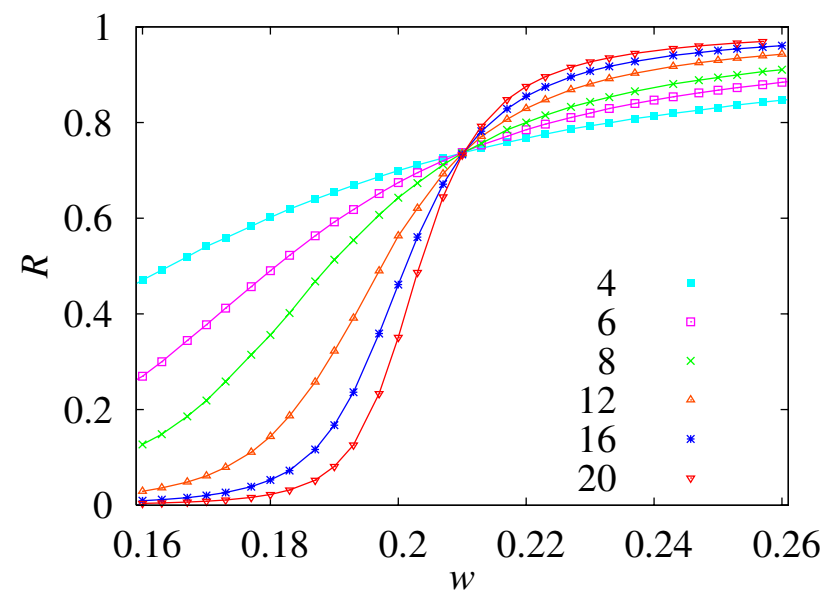

FIG. 5 (color online). Coarse plot of $R$ vs $w$ for spanning forests in dimension $d=4$ and lattice sizes $4 \leq L \leq 20$.
In Table I we summarize the estimated critical exponents for ferromagnetic Potts models with $q=0$ (this work), 1 (percolation), and 2 (Ising) in dimensions $d=$ $2,3,4,5$. It is evident that $\nu$ varies quite sharply as a function of $q$ and $d$, while $\gamma / \nu$ varies much more slowly. The dependences on dimension of $\nu$ and $\gamma / \nu$ for $q=0$ are consistent with the conjecture that they are tending to the mean-field values $1 / 2$ and 2 in dimension $d=6$, just as they do for $q=1$. This in turn supports the more general conjecture that the upper critical dimension is 6 for all random-cluster models with $0 \leq q<2$, and is 4 only when $q=2$.

This conjecture is supported by a field-theoretic renormalization-group calculation in dimension $d=6-$ $\epsilon$ through order $\epsilon^{3}$ [26] in which $q=2$ plays a distinguished role (all the correction terms vanish there). Specializing to $q=0$, we have

$$
\begin{aligned}
\gamma / \nu= & 2+\frac{\epsilon}{15}+\frac{7 \epsilon^{2}}{225}-\left(\frac{26 \zeta(3)}{625}-\frac{269}{16875}\right) \epsilon^{3}+O\left(\epsilon^{4}\right) \\
= & 2+0.066667 \epsilon+0.031111 \epsilon^{2}-0.034065 \epsilon^{3} \\
& +O\left(\epsilon^{4}\right) \\
1 / \nu= & 2-\frac{\epsilon}{3}-\frac{\epsilon^{2}}{30}+\left(\frac{4 \zeta(3)}{125}-\frac{173}{27000}\right) \epsilon^{3}+O\left(\epsilon^{4}\right) \\
= & 2-0.333333 \epsilon-0.033333 \epsilon^{2}+0.032058 \epsilon^{3} \\
& +O\left(\epsilon^{4}\right)
\end{aligned}
$$

These series seem rather difficult to resum, especially when $\epsilon \geq 2$, but they are in qualitative agreement with the exponents listed in Table I. Moreover, a slightly better agreement can be obtained by imposing the known exact values at $\epsilon=4$ on the interpolating function.

Details of these simulations and their data analysis, including analysis of universal amplitude ratios other than $R$, will be reported separately [19].

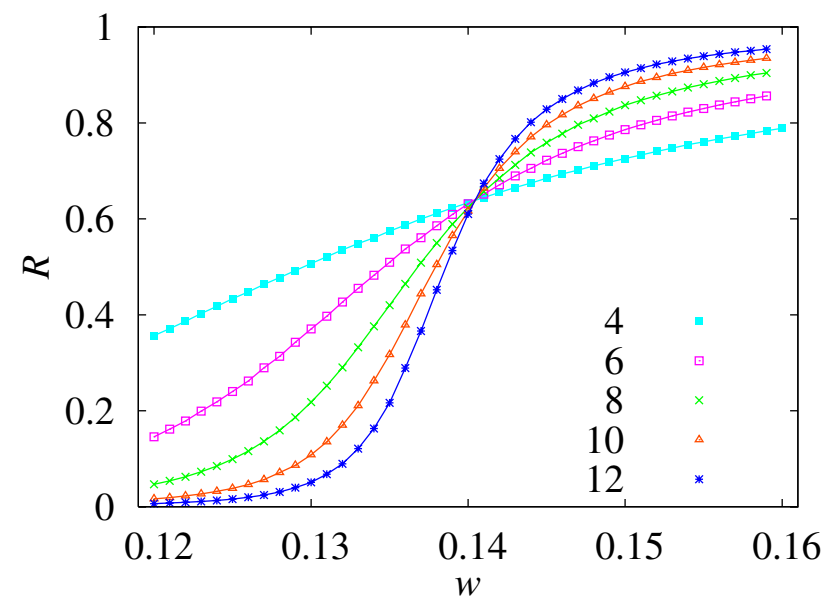

FIG. 6 (color online). Coarse plot of $R$ vs $w$ for spanning forests in dimension $d=5$ and lattice sizes $4 \leq L \leq 12$. 
TABLE I. Critical exponents $\nu$ and $\gamma / \nu$ vs $q$ and $d . d=2$ : presumed exact values [20]. $d=3$, 4, 5, $q=0$ : this work. $d=3, q=1:[21] . d=3, q=2:[22] . d=4, q=1:[23,24] . d=5$, $q=1:[24,25] . d=4,5, q=2$ : presumed exact values.

\begin{tabular}{|c|c|c|c|}
\hline & $q=0$ & $q=1$ & $q=2$ \\
\hline \multirow[t]{2}{*}{$d=2$} & $\nu=\infty$ & $\nu=4 / 3$ & $\nu=1$ \\
\hline & $\gamma / \nu=2$ & $\gamma / \nu=43 / 24$ & $\gamma / \nu=7 / 4$ \\
\hline \multirow{2}{*}{$d=3$} & $\nu=1.28(4)$ & $\nu=0.874(2)$ & $\nu=0.6301(5)$ \\
\hline & $\gamma / \nu=2.1675(10)$ & $\gamma / \nu=2.0455(6)$ & $\gamma / \nu=1.9634(5)$ \\
\hline \multirow[t]{2}{*}{$d=4$} & $\nu=0.80(1)$ & $\nu=0.689(10)$ & $\nu=1 / 2(\log )$ \\
\hline & $\gamma / \nu=2.1603(10)$ & $\gamma / \nu=2.094(3)$ & $\gamma / \nu=2(\log )$ \\
\hline \multirow[t]{2}{*}{$d=5$} & $\nu=0.59(2)$ & $\nu=0.57(1)$ & $\nu=1 / 2$ \\
\hline & $\gamma / \nu=2.08(2)$ & $\gamma / \nu=2.08(2)$ & $\gamma / \nu=2$ \\
\hline
\end{tabular}

We thank Henk Blöte, Sergio Caracciolo, Andrea Pelissetto, Jesús Salas, and Andrea Sportiello for helpful discussions. This work was supported in part by NSF Grants No. PHY-0116590 and No. PHY-0424082.

*Electronic address: yd10@nyu.edu

†Electronic address: garoni@nyu.edu

†Electronic address: sokal@nyu.edu

[1] R. B. Potts, Proc. Cambridge Philos. Soc. 48, 106 (1952).

[2] F. Y. Wu, Rev. Mod. Phys. 54, 235 (1982); 55, 315(E) (1983); J. Appl. Phys. 55, 2421 (1984).

[3] P.W. Kasteleyn and C. M. Fortuin, J. Phys. Soc. Jpn. Suppl. 26, 11 (1969); C. M. Fortuin and P. W. Kasteleyn, Physica (Utrecht) 57, 536 (1972).

[4] G. Grimmett, The Random-Cluster Model (SpringerVerlag, New York, 2006).

[5] R. J. Baxter, Exactly Solved Models in Statistical Mechanics (Academic Press, London, 1982).

[6] B. Nienhuis, J. Stat. Phys. 34, 731 (1984).

[7] P. Di Francesco, P. Mathieu, and D. Sénéchal, Conformal Field Theory (Springer-Verlag, New York, 1997).

[8] We stress that this is a lattice-dependent question; the answer is not universal among lattices of a given dimension $d$. However, for each spatial dimension $d$ one can also ask what is the maximum $q$ for which there exists a local Hamiltonian yielding a second-order transition: let us call this $q_{\star}(d)$. It is believed on the basis of conformal field theory that $q_{\star}(2)=4$, and it is generally expected that $q_{\star}(d)=2$ for $d \geq 4$ [A. Aharony and E. Pytte, Phys. Rev. B 23, 362 (1981); K. E. Newman, E. K. Riedel, and S. Muto, Phys. Rev. B 29, 302 (1984); 30, 2924(E) (1984)]. Little is known about the value of $q_{\star}(3)$. For further discussion, see H. Blöte, Y. Deng, X. Qian, and A.D. Sokal (to be published).

[9] M. J. Stephen, Phys. Lett. 56A, 149 (1976); F. Y. Wu, J. Phys. A 10, L113 (1977); J. L. Jacobsen, J. Salas, and A. D. Sokal, J. Stat. Phys. 119, 1153 (2005); A. D. Sokal, in Surveys in Combinatorics, 2005, edited by B.S. Webb (Cambridge University Press, Cambridge, England, 2005).

[10] S. Caracciolo, J. L. Jacobsen, H. Saleur, A. D. Sokal, and A. Sportiello, Phys. Rev. Lett. 93, 080601 (2004); see also
J.L. Jacobsen and H. Saleur, Nucl. Phys. B716, 439 (2005); S. Caracciolo, A. D. Sokal, and A. Sportiello (to be published).

[11] G. Kirchhoff, Ann. Phys. Chem. 72, 497 (1847); R. L. Brooks, C. A. B. Smith, A. H. Stone, and W. T. Tutte, Duke Math. J. 7, 312 (1940); A. Nerode and H. Shank, Am. Math. Mon. 68, 244 (1961); S. Chaiken, SIAM J. Algebr. Discrete Methods 3, 319 (1982); J. W. Moon, Discrete Math. 124, 163 (1994); A. Abdesselam, Adv. Appl. Math. 33, 51 (2004).

[12] F. Y. Wu, Phys. Rev. B 18, 516 (1978).

[13] L. Chayes and J. Machta, Physica (Amsterdam) A254, 477 (1998); see also Y. Deng, T. M. Garoni, J. Machta, and A. D. Sokal (to be published).

[14] R. H. Swendsen and J.-S. Wang, Phys. Rev. Lett. 58, 86 (1987); see also R. G. Edwards and A.D. Sokal, Phys. Rev. D 38, 2009 (1988).

[15] M. Sweeny, Phys. Rev. B 27, 4445 (1983). For variants of the Sweeny algorithm, see F. Gliozzi, Phys. Rev. E 66, 016115 (2002), as corrected by J.-S. Wang, O. Kozan, and R. H. Swendsen, Phys. Rev. E 66, 057101 (2002).

[16] See last paragraph of X.-J. Li and A. D. Sokal, Phys. Rev. Lett. 63, 827 (1989).

[17] A.D. Sokal, in Functional Integration: Basics and Applications, edited by C. de Witt-Morette, P. Cartier, and A. Folacci (Plenum, New York, 1997).

[18] D. Alberts, G. Cattaneo, and G. F. Italiano, ACM J. Exp. Algorithmics 2, 5 (1997); M. R. Henzinger and V. King, J. ACM 46, 502 (1999); R. D. Iyer, Jr. et al., ACM J. Exp. Algorithmics 6, 4 (2001); J. Holm, K. de Lichtenberg, and M. Thorup, J. ACM 48, 723 (2001).

[19] Y. Deng, T. M. Garoni, and A. D. Sokal (to be published).

[20] See, e.g., J. Salas and A. D. Sokal, J. Stat. Phys. 88, 567 (1997), Appendix A.1 and references cited therein.

[21] H. G. Ballesteros et al., J. Phys. A 32, 1 (1999); Y. Deng and H. W. J. Blöte, Phys. Rev. E 72, 016126 (2005).

[22] A. Pelissetto and E. Vicari, Phys. Rep. 368, 549 (2002); Y. Deng and H. W. J. Blöte, Phys. Rev. E 68, 036125 (2003).

[23] H. G. Ballesteros et al., Phys. Lett. B 400, 346 (1997).

[24] G. Paul, R. M. Ziff, and H. E. Stanley, Phys. Rev. E 64, 026115 (2001).

[25] J. Adler et al., Phys. Rev. B 41, 9183 (1990).

[26] O.F. de Alcantara Bonfim, J.E. Kirkham, and A.J. McKane, J. Phys. A 14, 2391 (1981). 